\title{
Coxiella burnetii Epitope-Specific T-Cell Responses in Patients with Chronic Q Fever
}

\author{
Anja Scholzen, ${ }^{a}$ Guilhem Richard, ${ }^{b}$ Leonard Moise,, ${ }^{b, c}$ Eva Hartman, ${ }^{a}$ Chantal P. Bleeker-Rovers, ${ }^{d}$ Patrick M. Reeves, ${ }^{e}$

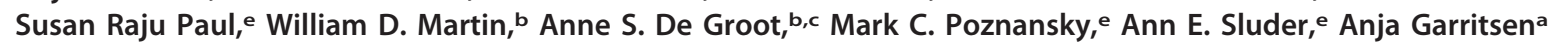

alnnatOss Laboratories B.V., Oss, the Netherlands

bEpiVax, Inc., Providence, Rhode Island, USA

Institute for Immunology and Informatics, Department of Cell and Molecular Biology, University of Rhode Island, Providence, Rhode Island, USA

dRadboud Expertise Center for Q Fever, Department of Internal Medicine, Radboud University Medical Center, Nijmegen, the Netherlands

eVaccine and Immunotherapy Center, Massachusetts General Hospital, Boston, Massachusetts, USA

ABSTRACT Infection with Coxiella burnetii, the causative agent of $\mathrm{Q}$ fever, can result in life-threatening persistent infection. Reactogenicity hinders worldwide implementation of the only licensed human $\mathrm{Q}$ fever vaccine. We previously demonstrated long-lived immunoreactivity in individuals with past symptomatic and asymptomatic Coxiella infection (convalescents) to promiscuous HLA class II C. burnetii epitopes, providing the basis for a novel T-cell targeted subunit vaccine. In this study, we investigated in a cohort of 22 individuals treated for persistent infection (chronic $\mathrm{Q}$ fever) whether they recognize the same set of epitopes or distinct epitopes that could be candidates for a therapeutic vaccine or aid in the diagnosis of persistent infection. In cultured enzyme-linked immunosorbent spot (ELISpot) assays, individuals with chronic Q fever showed strong class II epitope-specific responses that were largely overlapping with the peptide repertoire identified previously for convalescents. Five additional peptides were recognized more frequently by chronic subjects, but there was no combination of epitopes uniquely recognized by or nonreactive in subjects with chronic Q fever. Consistent with more recent/prolonged exposure, we found, however, stronger ex vivo responses by direct ELISpot to both whole-cell $C$. burnetii and individual peptides in chronic patients than in convalescents. In conclusion, we have validated and expanded a previously published set of candidate epitopes for a novel T-cell targeted subunit $\mathrm{Q}$ fever vaccine in treated patients with chronic $\mathrm{Q}$ fever and demonstrated that they successfully mounted a T-cell response comparable to that of convalescents. Finally, we demonstrated that individuals treated for chronic Q fever mount a broader ex vivo response to class II epitopes than convalescents, which could be explored for diagnostic purposes.

KEYWORDS Coxiella, ELISpot, Q fever, T cell, chronic, epitope, infection, peptide

fever is a zoonotic disease that is endemic in many countries worldwide. It is caused by the environmentally highly stable small Gram-negative coccobacillus Coxiella burnetii, which is transmitted to humans predominantly by aerosol from infected ruminants such as goats, sheep, and cattle (1). Outbreaks usually occur in the occupational setting, including among those in the livestock industry and deployed military personnel (1). Coxiella outbreaks can also occur in the general population, the largest to date being the outbreak in the Netherlands from 2007 to 2010, with an estimated 40,000 infections at the center of the epidemic area alone (2). While infection remains asymptomatic in an estimated $50 \%$ to $60 \%$ of individuals and acute symptomatic infection is readily treatable with antibiotics, a large proportion (10\% to $20 \%$ ) of individuals with acute $\mathrm{Q}$ fever later develop $\mathrm{Q}$ fever fatigue syndrome. Further, $1 \%$ to
Citation Scholzen A, Richard G, Moise L, Hartman E, Bleeker-Rovers CP, Reeves PM, Raju Paul S, Martin WD, De Groot AS, Poznansky MC, Sluder AE, Garritsen A. 2019. Coxiella burnetii epitope-specific T-cell responses in patients with chronic $\mathrm{Q}$ fever. Infect Immun 87:e00213-19. https://doi.org/ 10.1128/IAl.00213-19.

Editor Craig R. Roy, Yale University School of Medicine

Copyright $\odot 2019$ American Society for Microbiology. All Rights Reserved.

Address correspondence to Ann E. Sluder asluder@mgh.harvard.edu, or Anja Garritsen, anja.garritsen@innatoss.com.

A.E.S. and A.G. contributed equally to this article.

Received 18 March 2019

Returned for modification 24 June 2019

Accepted 17 July 2019

Accepted manuscript posted online 22 July 2019

Published 19 September 2019 
$5 \%$ of (often asymptomatically) infected individuals progress to persistent infection, also known as chronic Q fever. Chronic Q fever has a poor prognosis and manifests as endocarditis, infected aneurysms, or vascular prosthesis infection in individuals with specific risk factors $(1,3)$.

While $\mathrm{Q}$ fever infection in humans can be prevented by vaccination using Q-VAX, an inactivated whole-cell vaccine based on phase I C. burnetii, this vaccine is licensed for use in Australia only. Importantly, this vaccine requires prevaccination screening for prior exposure due to reported side effects in previously exposed individuals (3-5). In this context, the objective of the Q-VaxCelerate consortium is to develop a novel nonreactogenic T-cell-targeted human $\mathrm{Q}$ fever vaccine that does not require prescreening of vaccinees, rationally selecting T-cell epitopes for inclusion in such a vaccine $(5$, 6). Using immunoinformatically predicted T-cell epitopes derived from C. burnetii seroreactive and type IV secretion system (T4SS) substrate proteins, we previously analyzed antigenicity in naturally infected subjects with past symptomatic or asymptomatic C. burnetii infection; these are referred to as "convalescents" since infection was cleared. In these naturally exposed subjects, we demonstrated long-lived immunoreactivity to promiscuous CD4 T-cell epitopes, while HLA class I epitope responses were sparse in this cohort (7). One possible explanation for the latter finding was that class I responses might have contracted faster than class II responses, as previously observed following smallpox infection or vaccination and tuberculosis treatment (8-11). In this initial study, there were no striking differences between past asymptomatic or symptomatic infected individuals, all of whom successfully cleared acute $C$. burnetii infection. The question remains, however, as to whether, analogous to herpes simplex virus infection, there might be distinct epitope-specific T-cell repertoires for individuals that either successfully control infection or develop persistent infection (12). Such epitopes might be interesting targets for a potentially separate therapeutic vaccine to accelerate bacterial clearance in chronic $\mathrm{Q}$ fever or aid in the diagnosis of this persistent infection.

In the present study, we therefore analyzed T-cell reactivity to the same set of epitopes in a cohort of subjects diagnosed with and treated for persistent C. burnetii infection (chronic $\mathrm{Q}$ fever). The aim was to investigate whether subjects with chronic $\mathrm{Q}$ fever (i) show potentially greater reactivity to class I epitopes given their more recent exposure, (ii) recognize the same or a distinct set of class II epitopes, and (iii) differ in their effector memory T-cell response profile from individuals with resolved acute symptomatic or past asymptomatic infection.

\section{RESULTS}

Treated subjects with chronic $Q$ fever have cultured enzyme-linked immunosorbent spot assay (ELISpot) response patterns to HLA class I and II C. burnetii epitopes comparable to those of convalescent subjects. A group of 22 individuals with proven $(n=16)$ and probable $(n=6)$ chronic $Q$ fever consented for participation in this study (Table 1). All but two subjects with chronic Q fever still had phase I IgG titers of $\geq 1,024$ at inclusion in the study \{median (interquartile range [IQR]), 4,096 $(1,536$ to 8,192$)$, and $13 / 16$ subjects with proven chronic $Q$ fever and $1 / 6$ subjects with probable chronic $Q$ fever were still undergoing antibiotic treatment. For analysis of T-cell epitope-specific responses in the chronic $\mathrm{Q}$ fever cohort, preference was given to individuals with proven chronic Q fever who were diagnosed in 2016 or later and still undergoing antibiotic treatment. Subjects were scheduled for blood collection based on availability for class I and II peptide screening (Table 1). In total, 13 patients with proven chronic $\mathrm{Q}$ fever and 1 with probable chronic $\mathrm{Q}$ fever were tested for promiscuous class II epitope-specific responses (see Table S1 in the supplemental material) and 10 with proven and 3 with probable chronic $Q$ fever for class I epitope-specific responses (Table S2). HLA typing of the two selected groups showed supertype distributions largely comparable to expected frequencies in the general population and/or those in the previously analyzed convalescent groups (Table S3 and S4), except for an underrepresentation of HLA-DR11, -A11, and -B8 and an overrepresentation of HLA-A3 supertypes, which may be partially attributed to the small group sizes. 


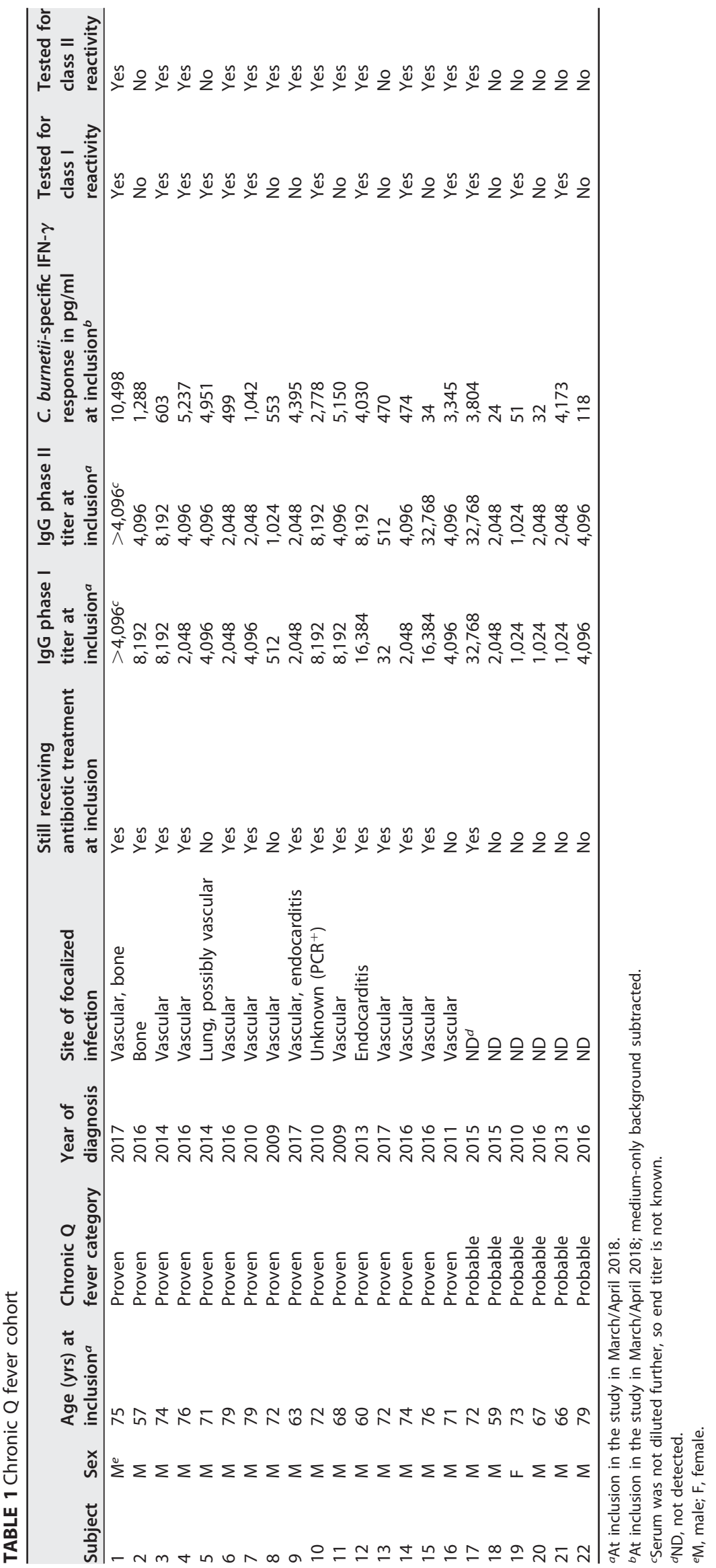


A

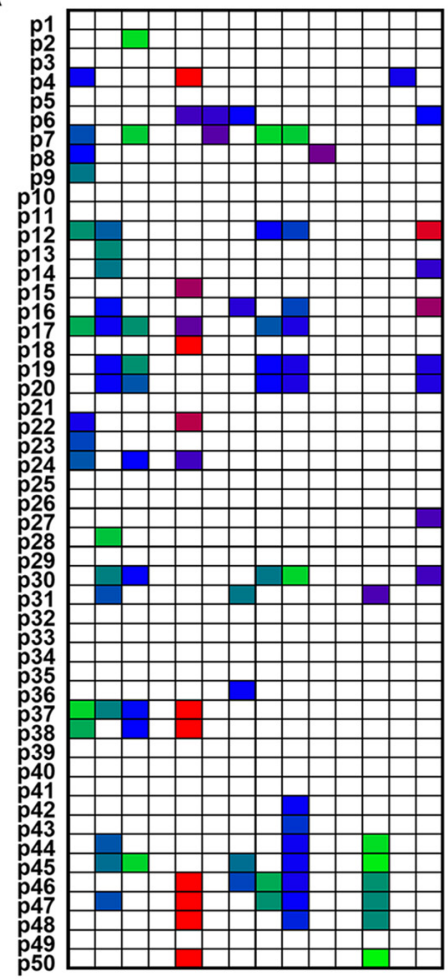

\section{B}

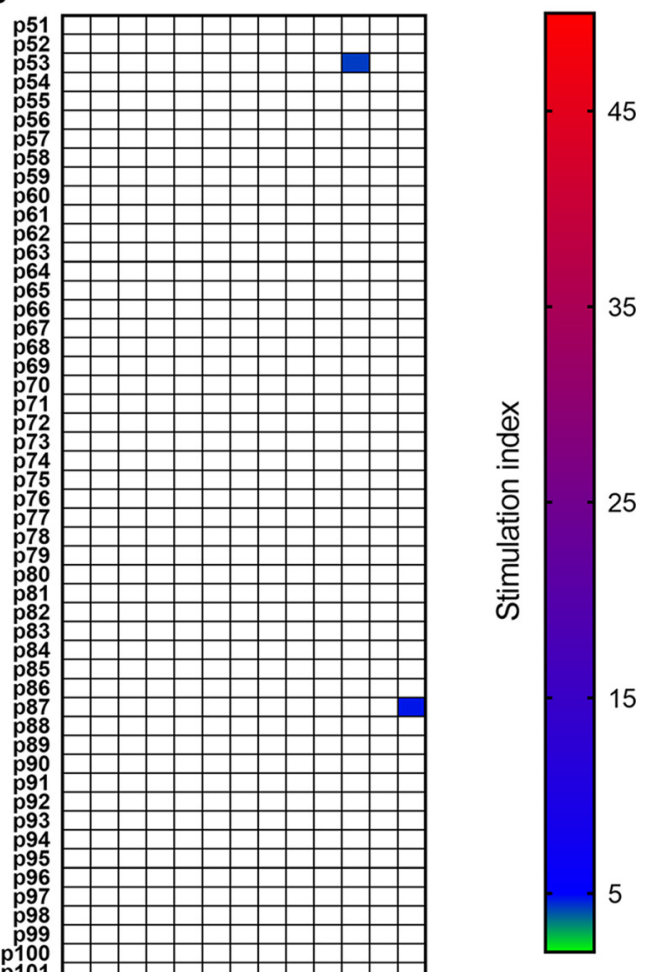

FIG 1 Cultured ELISpot human IFN- $\gamma$ responses to HLA class I and II peptides in individuals treated for chronic Q fever. Individual IFN- $\gamma$ responses to HLA class II (A) and class I (B) peptides determined by cultured ELISpot are depicted as stimulation indices (SI). Each column shows data from one donor, and each row shows responses to one of the 50 class II or 65 class I peptides. Responses not significantly different from background and/or lower than an average of 10 spots/well and/or an SI of $<2$ are shown as blanks. Significant responses with $\mathrm{SI} \geq 2$ are color coded as per the heat map key. Responses for one donor were capped at an SI of 50 to be able to properly resolve the magnitude of responses of the remaining subjects.

T-cell responses were first analyzed by cultured gamma interferon (IFN- $\gamma$ ) ELISpot, which both enhances detection of low-frequency responses and preferentially measures central memory T cells (13). Similar to previous observations for convalescent $C$. burnetii-exposed subjects, 9/14 patients with chronic $Q$ fever (64\%) showed responses to 3 to $14 \mathrm{HLA}$ class II peptides per donor (Fig. 1A), while responses to HLA class I peptides were rare, with only 3 subjects showing responses to 1 or 2 peptides each (Fig. 1B). When directly comparing the data from the chronic $Q$ fever cohort to those from convalescent subjects (asymptomatic, $n=33$, and symptomatic, $n=23$ ), there was no statistically significant difference in the breadth of the class II response per subject between either all chronic and all convalescent subjects $(P=0.15$ by Mann-Whitney test) or chronic subjects on the one hand and convalescent symptomatic or asymptomatic subjects on the other hand $(P=0.90$ and $P=0.16$ by Kruskal-Wallis test with Dunn's multiple-comparison post hoc test) (Fig. 2A). Nevertheless, chronic subjects had the smallest proportion of nonresponders among the three groups (Fig. 2B).

Both the overall breadth of responses and the responses to individual class II peptides largely overlapped between chronic and convalescent subjects. In total, 33/50 HLA class II peptides were recognized by at least $1 / 14$ chronic subjects, comparable to 
A

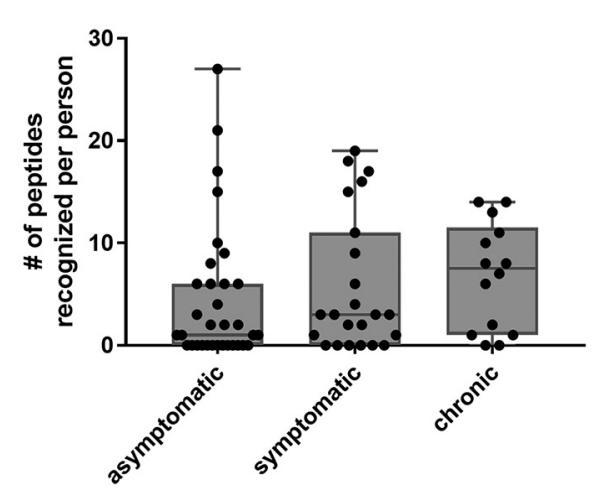

B convalescent (asymptomatic) convalescent (symptomatic)
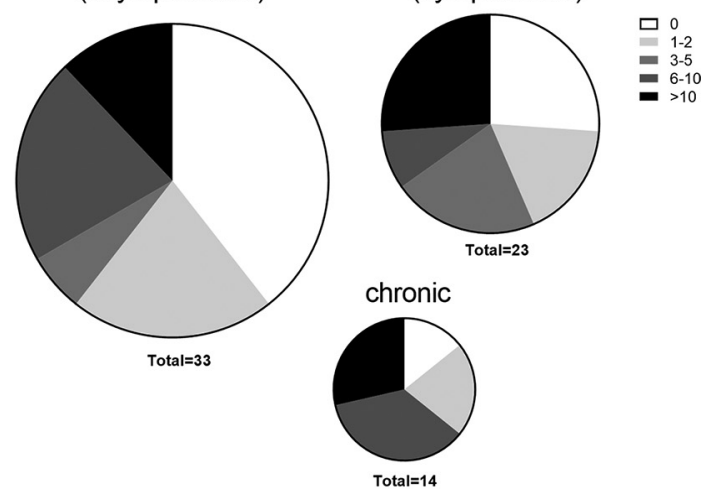

FIG 2 Cumulative HLA class II peptide responses in chronic compared to convalescent individuals. Data are shown for past asymptomatic $(n=33)$ or symptomatic $(n=23)$ infected individuals and for subjects with chronic $Q$ fever $(n=14)$ as the cumulative peptide response $(S I \geq 2)$ per donor $(A)$ or as the proportion of subjects recognizing 0,1 or 2,3 to 5,6 to 10 , or $>10$ peptides (B). Whisker-dot plots show the median and interquartile range (25th and 75 th percentiles), with whiskers extending from minimun to maximum values.

the fraction (28/50) of HLA class II peptides recognized by a similar proportion (at least $4 / 56[7.14 \%]$ ) of convalescent individuals. The same 22 peptides were recognized in both cohorts by at least $7 \%$ of the subjects. The peptides that were not recognized by any individual in the chronic cohort included 5/6 peptides that were also not recognized by any convalescent subject (p11, p34, p35, p40, and p49 [Fig. 3]).

More importantly, out of 21 highly antigenic HLA class II peptides that were previously found to be recognized by $>10 \%$ of all convalescent individuals (at least $6 / 56$ ), 15 were also recognized by $>10 \%$ of chronic subjects (at least $2 / 14$ and up to 6/14 [42\%] [Fig. 3]). This included at least one epitope from each of the five source proteins that were represented by two highly antigenic epitopes each in the convalescent cohort (p14 plus p15, CBU_1835/protoporphyrinogen oxidase; p18 plus p19, CBU_1513/protoporphyrinogen oxidase; p22 plus p23, CBU_1398/SucB; p37 plus p38, CBU_0718; and p45 plus p46, CBU_0307/outer membrane protein). Another 5 of these 21 highly antigenic peptides were recognized by $1 / 14$ chronic subjects. Only a single peptide that was highly antigenic in convalescents (p21 from CBU_1416/repressor protein (2) was not recognized by any chronic subject tested; however, 5/14 chronic individuals did recognize a second peptide (p2) from the same source protein.

Many of the class II peptide responses were at least as frequent in chronically infected subjects as in convalescents, and despite the large difference in group sizes, responses to six class II peptides were statistically significantly more frequent in chronic subjects (p6, p7, p16, p20, p30, and p47 [Fig. 3]). All of these six peptides were recognized by 4 or 5 individuals ( $28 \%$ to $36 \%$ ) within the chronic cohort, while for five of these peptides (all but p30), the frequency of responses in the convalescent cohort was $<10 \%$ ( 2 to 5 out of 56 subjects). However, for $3 / 5$ peptides to which $>10 \%$ of chronic subjects but $<10 \%$ of convalescents reacted, $>10 \%$ of convalescents did show a response to a second epitope from the same source protein. Finally, there was only a single peptide ( $p 9$, the single screened epitope from the hypothetical exported protein CBU_2065) that was recognized by one chronic subject but not a single individual in the convalescent cohort. Taken together, the results show that while (central memory) cultured ELISpot responses to some individual peptides are more frequent in chronic subjects, there is no strong evidence for a set of source proteins or epitopes for which responses are uniquely present or absent in subjects with chronic Q fever.

Treated subjects with chronic $Q$ fever show more frequent direct ELISpot responses to HLA class II C. burnetii epitopes than convalescent subjects. Given that subjects treated for chronic $\mathrm{Q}$ fever had a more recent and prolonged exposure to 
A

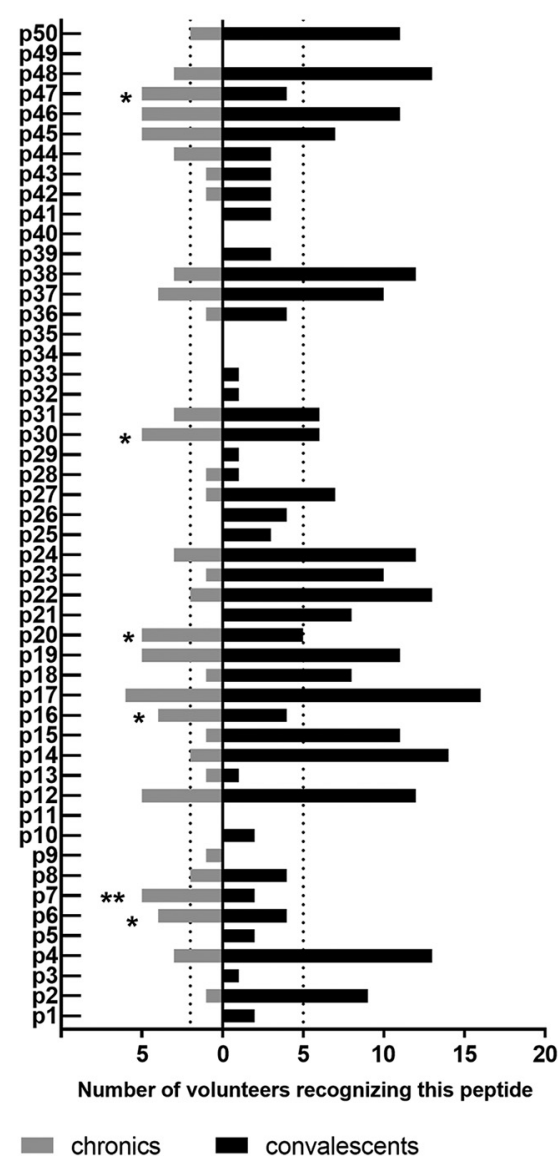

B

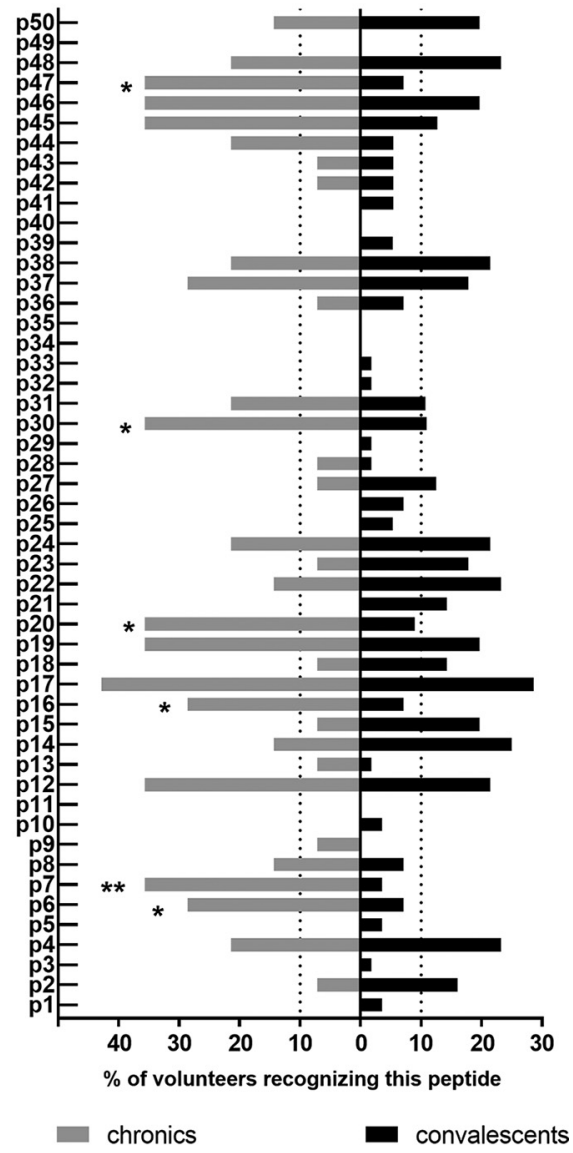

FIG 3 Class II peptide antigenicity patterns in chronic and convalescent individuals. Data are shown as the number (A) and proportion (B) of individuals with IFN- $\gamma$ responses to the 50 individual peptides in the chronic $(n=14)$ and convalescent cohorts (both past asymptomatic and symptomatic; $n=56$ ), as determined by cultured ELISpot. Bars extending over dotted lines indicate those peptides that were recognized by more than $10 \%$ of chronic $(\geq 2 / 14)$ or convalescent $(>5 / 56)$ subjects. Asterisks indicate significant difference in proportion between the two groups by Fisher's exact test. ${ }^{*}, P<0.05 ;{ }^{*}, P<0.01$.

C. burnetii, we hypothesized that these individuals might also show a stronger effector memory $T$-cell response profile than that of individuals with resolved acute $Q$ fever or past asymptomatic infection. Indeed, the individuals with chronic $Q$ fever enrolled in this study showed significantly higher IFN- $\gamma$ secretion measured following whole-blood stimulation with heat-killed whole-cell C. burnetii (strain Cb02629) than did convalescent subjects (Table 1 and Fig. S1A). Stimulation of freshly isolated peripheral blood mononuclear cells (PBMCs) for direct ELISpot with whole-cell C. burnetii indicated that this was at least partially due to a higher frequency of responding cells, with significantly higher numbers of spot-forming units (SFU) and higher stimulation indices in chronic than in convalescent subjects (Fig. S1B and C). Multiplex cytokine analysis of supernatants from whole-blood stimulation revealed that the greater ex vivo response was not confined to IFN- $\gamma$ but was also evident for interleukin 2 (IL-2) responses to whole-cell heat-killed C. burnetii (Fig. S2A). The ratio between IFN- $\gamma$ and IL-2 responses in chronic subjects did not differ from that found for convalescent subjects (Fig. S2A). IL-10 responses, in contrast, were lower in the chronic $Q$ fever cohort, and innate tumor necrosis factor alpha (TNF- $\alpha$ ) and IL-1 $\beta$ responses did not differ between chronic and convalescent subjects (Fig. S2B).

For a subset of chronic and convalescent individuals, we next analyzed by direct ELISpot whether HLA class II C. burnetii-specific peptide responses would also be more readily detected in chronic patients. This assay preferentially measures effector memory 
A

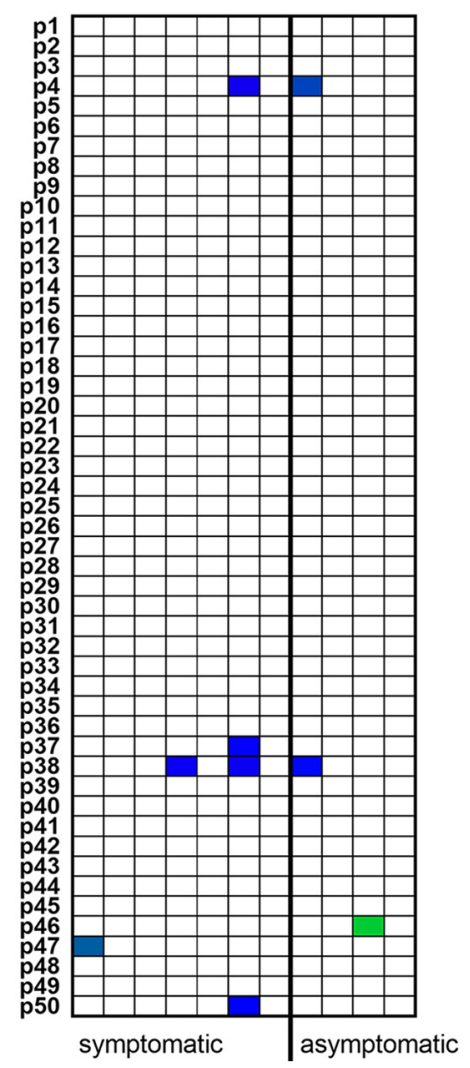

B

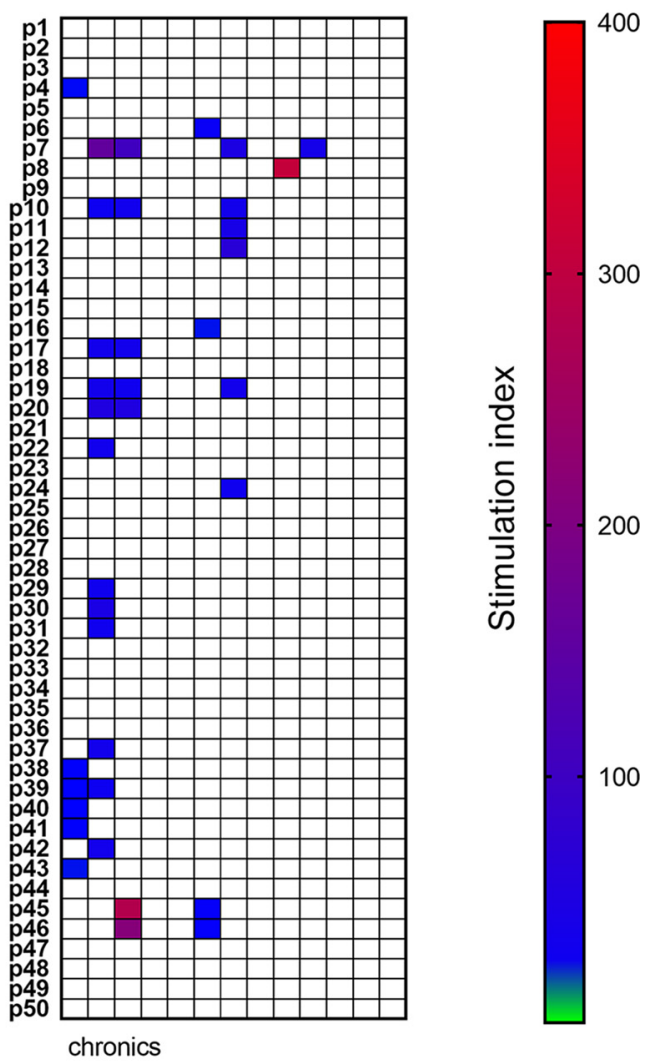

FIG 4 Direct ELISpot human IFN- $\gamma$ responses to HLA class II peptides. IFN- $\gamma$ responses to individual HLA class II peptides were determined by direct ELISpot for convalescent individuals with a history of symptomatic ( $n=7)$ or asymptomatic $(n=4)$ Q fever infection (A) and individuals with chronic Q fever $(n=13)$ (B). Data are depicted as SI for all subjects analyzed. Each column shows data from one donor, and each row shows responses to one of the 50 class II peptides. Responses not significantly different from background and/or lower than an average of 10 spots/million cells plated and/or an $\mathrm{SI}$ of $<2$ are shown as blanks. Significant responses $(\mathrm{SI} \geq 2)$ are color coded as per the heat map key.

responses (13). The proportions of individuals with direct ELISpot responses in the two cohorts were comparable, with 5/11 responding convalescent individuals and 7/13 chronic subjects (Fig. 4). However, the breadth of the response was larger for chronic subjects: one of the seven responding chronic subjects showed ex vivo responses to 4 class II peptides, and another four subjects scored positive for 6 to 12 class II peptides. In contrast, only one of the responding convalescent subjects recognized four peptides and the remaining three individuals only one or two peptides. All but one chronic subject and all convalescent individuals with responses detectable by direct ELISpot also showed responses by cultured ELISpot, and the individual peptides recognized in both assays per donor largely overlapped between these two groups (Fig. S3 and S4). Although there were three peptides that elicited direct recall responses with a relatively high proportion of individuals exclusively in the chronic group (p7 in 4/13 and p10 and p19 in 3/13), this difference did not reach statistical significance by Fisher's exact test, given the small number $(n=11)$ of convalescent subjects also tested. Among convalescent subjects, the only peptides recognized by more than one individual were p4 and p38, two of the highly reactive peptides in the cultured ELISpot assay.

\section{DISCUSSION}

In this study, we compared the repertoire of HLA class II T-cell epitopes recognized by subjects treated for persistent $C$. burnetii infection (chronic $Q$ fever) with that recognized by convalescent individuals (i.e., those with resolved past acute or asymp- 
tomatic infection) in our previous study (7). We found that individuals treated for chronic $\mathrm{Q}$ fever effectively generated a central memory $C$. burnetii-specific T-cell response, as measured by cultured ELISpot, that closely resembles that of convalescent patients. This includes both the breadth and the individual HLA class II epitopes recognized and the near absence of detectable responses to HLA class I peptides. The main differences between the two cohorts were that treated subjects with chronic $Q$ fever showed a higher proportion of cultured ELISpot responses to a small subset of six promiscuous CD4 T-cell epitopes than did convalescents and exhibited detectable effector memory responses, as measured by direct ELISpot, to a greater number of peptides per subject. Both are consistent with more recent and prolonged antigen exposure in the subjects with chronic Q fever.

Our study provides validation of the high antigenic potential of the previously identified 22 promiscuous HLA class II peptides and the identification of an additional five C. burnetii HLA class II epitopes (p6, p7, p16, p20, and p47). These five peptides were recognized by four or five individuals ( $28 \%$ to $36 \%$ ) within the chronic cohort, highlighting their strong antigenic potential at least during or shortly after infection. Bearing in mind the small size of the group of chronic patients evaluated, the fact that all but one of the epitopes found to be highly antigenic in convalescent subjects (recognized by $>10 \%$ individuals) were also recognized by at least one subject in the chronic cohort further indicates that at least among this set of screened HLA class II epitopes, there is no unique set of peptides to which responses would be absent in persistently infected individuals and that would warrant consideration for a separate therapeutic vaccine for chronic $\mathrm{Q}$ fever patients. These results for chronic $\mathrm{Q}$ fever are in contrast to the observation of "asymptomatic" epitopes in herpes simplex virus infection (12). The principal difference may be that unlike $Q$ fever, herpes simplex virus infection is always considered a persistent infection but can remain asymptomatic nonetheless. Instead, the same set of promiscuous HLA class II epitopes identified previously in the convalescent cohort (7) could in principle be used to further boost already primed T-cell responses in individuals with persistent infection. Whether this would speed up resolution of infection in this patient group, however, is unclear. Evidently, the IFN- $\gamma$ recall response of circulating $\mathrm{T}$ cells in individuals with chronic $\mathrm{Q}$ fever is fully functional, both in response to individual epitopes and in response to whole-cell $C$. burnetii. This is in line with previous studies using IFN- $\gamma$ enzyme-linked immunosorbent assay (ELISA) following whole-blood stimulation $(14,15)$ and ELISpot using freshly isolated PBMCs (16). If this strong IFN- $\gamma$ response is insufficient to promote clearance of infection foci by activating C. burnetii-infected monocytes/macrophages, then the defect could be downstream of IFN- $\gamma$ signaling, as proposed previously (14). In particular, antigen-presenting-cell maturation, function, and interaction with $\mathrm{T}$ cells as mediated via the IFN- $\gamma$-IL-12p40 feedback loop (14, 17-19) in foci of infection could be compromised in persistent infection. Therefore, further research is required to clarify whether patients with chronic Q fever can benefit from a therapeutic vaccine or whether a completely different approach is needed to achieve clearance in this population.

The results of this study indicate that the unexpected scarcity of detectable responses to the predicted HLA class I epitopes in convalescent subjects (7) is not simply due to the long interval between initial exposure and T-cell assays in our previous study, given that the chronic subjects analyzed in this investigation were exposed to $C$. burnetii antigens until much more recently. An obvious potential confounder in this chronic $\mathrm{Q}$ fever cohort is the fact that these subjects had been diagnosed and received antibiotic treatment for various lengths of time. Heterogeneity in time since diagnosis and in the duration of ongoing treatment, however, was minimized during selection of individuals for epitope screening. Moreover, a previous study showed that duration of antibiotic treatment following diagnosis of chronic $Q$ fever, and whether subjects received treatment or not, did not influence IFN- $\gamma$ secretion, at least not in a wholeblood stimulation assay using whole-cell C. burnetii (14). Of note, CD8 responses have been shown to decline rapidly following Mycobacterium tuberculosis treatment $(10,11)$, 
and we cannot exclude that this might have also impacted class I responses in the present cohort. The question of whether and which class I epitopes should be included in a T-cell targeted $\mathrm{Q}$ fever vaccine for humans therefore requires further investigation during a new outbreak or a vaccination campaign.

In line with the observed higher frequency of circulating effector $T$ cells responding directly ex vivo to C. burnetii epitopes as well as whole-cell C. burnetii in these persistently infected individuals, we found not only stronger IFN- $\gamma$ responses but also significantly higher IL-2 production in chronic than in convalescent individuals. IL-2 is mainly produced by antigen-specific activated CD4 T cells shortly after T-cell receptor engagement (20). Of note, these results contrast with those of a previous study that found lower IL-2 secretion in response to whole-cell $C$. burnetii and an elevated IFN- $\gamma /$ IL-2 ratio in patients with chronic $\mathrm{Q}$ fever. This finding was hypothesized to reflect increased numbers of circulating effector T cells producing IFN- $\gamma$ and small amounts of IL-2 (14). However, a simple supernatant secretion assay does not distinguish whether different cytokines are produced by the same or different cell populations, and central memory T cells can also coproduce two or more cytokines, including IL-2 (21). A possible technical explanation for the discrepancy between the two studies in regard to IL-2 secretion and the IFN- $\gamma / \mathrm{IL}-2$ ratio is that in the previous study, IL-2 responses were assessed after $48 \mathrm{~h}$ rather than $24 \mathrm{~h}$ of stimulation (14), which may have impacted measurement of this rapidly consumed growth factor. Moreover, while individuals in both studies were recruited from the same region and all were likely initially exposed during the 2007-2011 outbreak, patients for the other study were assessed approximately 5 years earlier. Thus, cellular responses assessed in this investigation have likely further contracted in convalescents since their initial exposure, potentially to different degrees for each cytokine. Infection and thus antigen exposure in chronic patients, in contrast, were persistent and hence longer. Whether the longer clinical prepatency in our cohort might potentially relate to stronger IL-2 responses can only be speculated on and requires investigation in a larger, specifically designed study.

Diagnosis of chronic Q fever through culture or PCR-based detection of bacteria from the infected tissue is not always possible, and an array of additional methods, including imaging and serology, as well as clinical risk factors is used to guide clinical decision-making and treatment (22). However, high antibody titers toward phase I C. burnetii, used as one key risk criterion, are also found in a significant proportion of occupationally exposed individuals or past acute cases (23). Therefore, more discriminative immune readouts would be desirable to aid the diagnosis of chronic $\mathrm{Q}$ fever. One obvious question arising from this limited data set is whether direct ex vivo responses to specific epitopes such as $\mathrm{p} 7, \mathrm{p} 10$, and p19, which were confined to the chronic cohort, might be of diagnostic value. This would require evaluation of a much larger cohort of previously exposed individuals and (ideally recently diagnosed) chronic $Q$ fever cases and a separate group of subjects with acute $Q$ fever or recently recovered individuals. Only then will it be possible to determine whether ex vivo responses to these epitopes correlate with persistent infection or simply with recent exposure. To be of value as a diagnostic tool, coverage of subjects would have to be greater than the currently observed $23 \%$ to $30 \%$. Otherwise, the assay would at best be of supporting value in addition to the existing set of PCR, serology, and scanning techniques to localize infection.

In conclusion, we here validate and expand the characterization of a previously published set of promiscuous C. burnetii-specific HLA class II T-cell epitope clusters as candidates for a novel T-cell targeted subunit $\mathrm{Q}$ fever vaccine. We found that treated patients with chronic $Q$ fever mounted a central memory recall T-cell response comparable to that of convalescent individuals. Finally, we demonstrate that individuals treated for chronic $\mathrm{Q}$ fever mount a broader ex vivo response to class II epitopes, which could be explored for diagnostic purposes. 


\section{MATERIALS AND METHODS}

Study population. Twenty-two participants were recruited who were receiving or had received treatment for chronic Q fever or persistent focalized infection at the outpatient clinics of the Radboud University Medical Center in Nijmegen, the Elisabeth Hospital in Tilburg, the Jeroen Bosch Hospital in 's-Hertogenbosch, the Bernhoven Hospital in Uden, the Medisch Spectrum Twente hospital in Twente, the Medical University Centre in Maastricht, and the Zuyderland Medical Center in Heerlen, the Netherlands. The chronic Q fever group comprised 16 patients with proven and 6 patients with probable chronic Q fever diagnosed according to the Dutch consensus guideline on chronic Q fever (22). Ten of the patients with proven chronic $Q$ fever presented with a vascular focus in an aneurysm or aortic prosthesis, one with endocarditis, one with both a vascular and valvular localization, one with both vascular and vertebral foci, one with lesions in the lung and an aneurysm, one with a focus in vertebrae only, and one with a positive PCR. Thirteen of the patients with proven and one of the patients with probable cases were still on antibiotic treatment at the time of inclusion in this study (Table 1). At inclusion in the study, all participants treated for chronic $\mathrm{Q}$ fever completed a medical questionnaire and donated blood for HLA typing and analysis of serological and cellular responses to whole-cell C. burnetii.

C. burnetii-specific cultured ELISpot responses determined for this chronic $\mathrm{Q}$ fever cohort were compared to previously published results (7) from previously exposed individuals with a history of resolved asymptomatic or symptomatic $Q$ fever infection who were recruited from the village of Herpen, the Netherlands, one of the focal centers of the 2007-2010 Q fever outbreak (24, 25). Additional assays in the present study (direct ELISpot responses and cytokine release during whole-blood stimulation) were conducted using a subgroup from this cohort of convalescent $Q$ fever-exposed individuals from Herpen.

The study was reviewed and approved by the Medical Ethical Committee Brabant (Tilburg, Netherlands; NL51305.028.15), and all participants provided written informed consent.

HLA typing and serological and cellular responses to whole-cell $C$. burnetii at inclusion. HLA typing was performed at the HLA laboratory at the Laboratory of Translational Immunology at the UMC Utrecht, the Netherlands, by next-generation sequencing, and the resulting HLA-A, HLA-B, and HLA-DRB1 alleles were assigned to supertype families as described previously (7).

IgG and IgM antibody titers for phase I and phase II C. burnetii were determined by immunofluorescence assay (Focus Diagnostics) at the Jeroen Bosch Hospital, 's-Hertogenbosch, the Netherlands.

Cellular responses were determined by whole-blood IFN- $\gamma$ release assay (IGRA) (Q-detect), using lithium-heparin-anticoagulated blood stimulated with C. burnetii antigen (heat-killed Cb02629, lot 14VRIM014; Wageningen Bioveterinary Research) and appropriate positive and negative controls, as described previously (7). In addition to IFN- $\gamma$ ELISA, multiplex cytokine analysis of whole-blood stimulation supernatants for IFN- $\gamma$, IL-2, IL-10, TNF- $\alpha$, and IL- $1 \beta$ was conducted using the human proinflammatory panel $1 \mathrm{~V}$-Plex assay (Mesoscale Discovery) according to the manufacturer's recommendations. Of note, the V-Plex assay uses a different standard than the Q-detect ELISA, resulting in an approximately 20 -fold difference in calculated IFN- $\gamma$ concentrations.

Analysis of $\boldsymbol{C}$. burnetii epitope-specific T-cell responses. Antigen-specific T-cell responses to $C$. burnetii were determined by ELISpot using 50 broadly promiscuous HLA class II epitope clusters (Table S1) and 65 HLA class I epitopes (Table S2). As described previously (7), these 115 epitopes were derived by immunoinformatic prediction using the iVAX toolkit developed by EpiVax (http://epivax.com/ immunogenicity-screening/ivax-web-based-vaccine-design) $(26,27)$ from two sets of $C$. burnetii antigens: type IV secretion system (T4SS) substrates expected to elicit CD8 responses and known seroreactive $C$. burnetii antigens based on antibody responses in humans and mice.

Two different ELISpots were employed to facilitate detection of central memory T-cell responses (cultured ELISpot) and effector memory T-cell responses (standard or direct ELISpot) (13). ELISpot was conducted using freshly isolated peripheral blood mononuclear cells (PBMCs) from lithium-heparinanticoagulated blood, using Leukosep tubes prefilled with Ficoll (Greiner BioOne) according to the manufacturer's recommendations. ELISpots were conducted based on a published protocol, using MultiScreen IP filter plates (Merck Millipore) and a human IFN- $\gamma$ ELISPOT antibody and reagent set (Diaclone) to detect responses to individual peptides in quadruplicate (final concentration, $2 \mu \mathrm{g} / \mathrm{ml}$ per peptide; $0.02 \%$ dimethyl sulfoxide [DMSO]) (7). Plates were scanned on an AID Classic reader system, and spot-forming units were counted using AID ELISpot software v7.0 (both AID Diagnostika GmbH). Statistical analyses were carried out using GraphPad Prism software (v7).

For detection of $C$. burnetii-specific central memory T-cell responses and to increase sensitivity to low-frequency antigen-specific T cells, ELISpot was preceded by antigen-specific T-cell expansion with peptide pools (7). Based on cell availability, a median of 41,000 cells per expansion culture (interquartile range [IQR], 32,000 to 51,000) were plated per replicate well for subjects with chronic Q fever and data were analyzed as described previously using three combined threshold criteria (7): cultured ELISpot peptide restimulation responses were defined as positive when they (i) were significantly higher than spot counts in matched negative-control wells from the same expansion culture by one-way analysis of variance (ANOVA) with Holm-Šídák multiple-comparison correction post hoc test, (ii) reached a stimulation index of at least 2 above that of the matched negative-control wells, and (iii) reached an absolute cutoff of $>10$ SFU/well.

For direct ELISpot, epitope-specific HLA class II responses were evaluated ex vivo without prior culture or expansion for all chronic individuals with a sufficiently large number of PBMCs available $(n=13)$ and for a subset of convalescent individuals $(n=11)$. Due to the expected lower precursor frequency in fresh PBMCs and based on cell availability, a median of 215,000 cells (IQR, 115,000 to 331,000) were plated per replicate well for subjects with chronic $Q$ fever and convalescent subjects. In addition to peptide stimulation, duplicate wells of PBMCs were also stimulated with whole-cell heat-killed C. burnetii antigen 
(strain Cb02629) at the same concentration as used for whole-blood stimulations in Q-detect. The threshold criteria for a positive response in the direct ELISpot were (i) spot counts significantly higher than those in matched negative-control wells from the same donor by one-way ANOVA with Holm-Šídák multiple-comparison correction post hoc test, (ii) responses that reached a stimulation index of at least 2 above that of the matched negative-control wells, and (iii) an absolute cutoff of $10 \mathrm{SFU} / \mathrm{million}$ cells.

\section{SUPPLEMENTAL MATERIAL}

Supplemental material for this article may be found at https://doi.org/10.1128/IAI .00213-19.

SUPPLEMENTAL FILE 1, PDF file, 1.2 MB.

\section{ACKNOWLEDGMENTS}

We thank all patients with chronic $\mathrm{Q}$ fever as well as the volunteers from the village of Herpen, the Netherlands, for their participation in this study. We acknowledge the patient organizations Q-support and Q-uestion and the following physicians for their assistance in recruiting chronic $Q$ fever patients in this study: $M$ van Kasteren (ElisabethTweeSteden Hospital Tilburg) and A. Olde Loohuis. P. Hindocha is acknowledged for assistance with HLA supertype assignment.

This research was supported by contract HDTRA1-15-C-0020 from the U.S. Defense Threat Reduction Agency (https://www.dtra.mil), awarded to Massachusetts General Hospital (MGH; lead principal investigator, M.C.P.); work by authors at other institutions was supported by subcontracts under the prime contract award to $\mathrm{MGH}$.

The funder had no role in study design, data collection, analysis or interpretation of the data, the preparation of the manuscript, or the decision to submit the work for publication.

A.G. is a senior officer and shareholder and A.S. is an employee of Innatoss Laboratories B.V., which provides diagnostic screening for Q fever. A.S.D.G. and W.D.M. are senior officers and shareholders and L.M. and G.R. are employees of EpiVax, Inc., a company specializing in immunoinformatic analysis. Innatoss Laboratories B.V. and EpiVax, Inc., own patents to technologies utilized by associated authors in the research reported here. The remaining authors declare that the research was conducted in the absence of any commercial or financial relationships that could be construed as a potential conflict of interest.

A.S., P.M.R., M.C.P., A.E.S., and A.G. formulated research goals; A.S. and A.G. designed experiments; E.H. collected clinical data; C.P.B.-R. supported patient recruitment; S.R.P. advised on patient selection and reviewed clinical data; A.S. conducted experiments and analyzed data; A.G., W.D.M., and A.S.D.G. contributed vital reagents and computing tools; L.M. and G.R. performed immunoinformatic epitope predictions and selection; G.R. analyzed HLA supertypes; A.S. and A.G. interpreted the data and wrote the manuscript; and G.R., L.M., E.H., C.P.B.-R., P.M.R., S.R.P., W.D.M., A.S.D.G., M.C.P., and A.E.S. discussed data and critically revised the manuscript. All authors read and approved the final manuscript.

\section{REFERENCES}

1. Eldin C, Melenotte C, Mediannikov O, Ghigo E, Million M, Edouard S, Mege JL, Maurin M, Raoult D. 2017. From Q fever to Coxiella burnetii infection: a paradigm change. Clin Microbiol Rev 30:115-190. https://doi .org/10.1128/CMR.00045-16.

2. Kampschreur LM, Hagenaars JC, Wielders CC, Elsman P, Lestrade PJ, Koning OH, Oosterheert JJ, Renders NH, Wever PC. 2013. Screening for Coxiella burnetii seroprevalence in chronic $\mathrm{Q}$ fever high-risk groups reveals the magnitude of the Dutch $\mathrm{Q}$ fever outbreak. Epidemiol Infect 141:847-851. https://doi.org/10.1017/S0950268812001203.

3. Kampschreur LM, Oosterheert JJ, Hoepelman Al, Lestrade PJ, Renders $\mathrm{NH}$, Elsman P, Wever PC. 2012. Prevalence of chronic Q fever in patients with a history of cardiac valve surgery in an area where Coxiella burnetii is epidemic. Clin Vaccine Immunol 19:1165-1169. https://doi.org/10 .1128/CVI.00185-12.

4. Marmion BP, Ormsbee RA, Kyrkou M, Wright J, Worswick DA, Izzo AA, Esterman A, Feery B, Shapiro RA. 1990. Vaccine prophylaxis of abattoir-associated Q fever: eight years' experience in Australian abattoirs. Epidemiol Infect 104:275-287. https://doi.org/10.1017/ s0950268800059458.

5. Ruiz S, Wolfe DN. 2014. Vaccination against Q fever for biodefense and public health indications. Front Microbiol 5:726. https://doi.org/10.3389/ fmicb.2014.00726.

6. Reeves PM, Paul SR, Sluder AE, Brauns TA, Poznansky MC. 2017. Q-vaxcelerate: a distributed development approach for a new Coxiella burnetii vaccine. Hum Vaccin Immunother 13:2977-2981. https://doi .org/10.1080/21645515.2017.1371377.

7. Scholzen A, Richard G, Moise L, Baeten LA, Reeves PM, Martin WD Brauns TA, Boyle CM, Raju Paul S, Bucala R, Bowen RA, Garritsen A, De Groot AS, Sluder AE, Poznansky MC. 2019. Promiscuous Coxiella burnetii CD4 epitope clusters associated with human recall responses are candidates for a novel T-cell targeted multi-epitope $\mathrm{Q}$ fever vaccine. Front Immunol 10:207. https://doi.org/10.3389/fimmu.2019.00207. 
8. Amara RR, Nigam P, Sharma S, Liu J, Bostik V. 2004. Long-lived poxvirus immunity, robust CD4 help, and better persistence of CD4 than CD8 T cells. J Virol 78:3811-3816. https://doi.org/10.1128/jvi.78 .8.3811-3816.2004.

9. Hammarlund E, Lewis MW, Hanifin JM, Mori M, Koudelka CW, Slifka MK. 2010. Antiviral immunity following smallpox virus infection: a case-control study. J Virol 84:12754-12760. https://doi.org/10.1128/ JVI.01763-10.

10. Nyendak MR, Park B, Null MD, Baseke J, Swarbrick G, Mayanja-Kizza H, Nsereko M, Johnson DF, Gitta P, Okwera A, Goldberg S, Bozeman L, Johnson JL, Boom WH, Lewinsohn DA, Lewinsohn DM, Tuberculosis Research Unit, the Tuberculosis Trials Consortium. 2013. Mycobacterium tuberculosis specific CD8(+) T cells rapidly decline with antituberculosis treatment. PLoS One 8:e81564. https://doi.org/10.1371/journal.pone .0081564 .

11. Axelsson-Robertson R, Rao M, Loxton AG, Walzl G, Bates M, Zumla A, Maeurer M. 2015. Frequency of Mycobacterium tuberculosis-specific CD8 + T-cells in the course of anti-tuberculosis treatment. Int J Infect Dis 32:23-29. https://doi.org/10.1016/j.ijid.2015.01.017.

12. Chentoufi AA, Kritzer E, Yu DM, Nesburn AB, Benmohamed L. 2012. Towards a rational design of an asymptomatic clinical herpes vaccine: the old, the new, and the unknown. Clin Dev Immunol 2012:187585. https://doi.org/10.1155/2012/187585.

13. Calarota SA, Baldanti F. 2013. Enumeration and characterization of human memory $\mathrm{T}$ cells by enzyme-linked immunospot assays. Clin Dev Immunol 2013:637649. https://doi.org/10.1155/2013/637649.

14. Schoffelen T, Sprong T, Bleeker-Rovers CP, Wegdam-Blans MC, Ammerdorffer A, Pronk MJ, Soethoudt YE, van Kasteren ME, Herremans T, Bijlmer HA, Netea MG, van der Meer JW, Joosten LA, van Deuren M. 2014. A combination of interferon-gamma and interleukin-2 production by Coxiella burnetii-stimulated circulating cells discriminates between chronic Q fever and past Q fever. Clin Microbiol Infect 20:642-650. https://doi.org/10.1111/1469-0691.12423.

15. Schoffelen T, Textoris J, Bleeker-Rovers CP, Ben Amara A, van der Meer JW, Netea MG, Mege JL, van Deuren M, van de Vosse E. 2017. Intact interferon-gamma response against Coxiella burnetii by peripheral blood mononuclear cells in chronic Q fever. Clin Microbiol Infect 23: 209.e9-209.e15. https://doi.org/10.1016/j.cmi.2016.11.008.

16. Limonard GJ, Thijsen SF, Bossink AW, Asscheman A, Bouwman JJ. 2012. Developing a new clinical tool for diagnosing chronic $Q$ fever: the Coxiella ELISPOT. FEMS Immunol Med Microbiol 64:57-60. https://doi .org/10.1111/j.1574-695X.2011.00890.x.

17. Brodsky FM, Lem L, Solache A, Bennett EM. 1999. Human pathogen subversion of antigen presentation. Immunol Rev 168:199-215. https:// doi.org/10.1111/j.1600-065X.1999.tb01294.x.

18. Gorvel L, Ben Amara A, Ka MB, Textoris J, Gorvel JP, Mege JL. 2014.
Myeloid decidual dendritic cells and immunoregulation of pregnancy: defective responsiveness to Coxiella burnetii and Brucella abortus. Front Cell Infect Microbiol 4:179. https://doi.org/10.3389/fcimb.2014.00179.

19. Gorvel L, Textoris J, Banchereau R, Ben Amara A, Tantibhedhyangkul W, von Bargen K, Ka MB, Capo C, Ghigo E, Gorvel JP, Mege JL. 2014. Intracellular bacteria interfere with dendritic cell functions: role of the type I interferon pathway. PLoS One 9:e99420. https://doi.org/10.1371/ journal.pone.0099420.

20. Boyman O, Sprent J. 2012. The role of interleukin-2 during homeostasis and activation of the immune system. Nat Rev Immunol 12:180-190. https://doi.org/10.1038/nri3156.

21. Mahnke YD, Brodie TM, Sallusto F, Roederer M, Lugli E. 2013. The who's who of T-cell differentiation: human memory T-cell subsets. Eur J Immunol 43:2797-2809. https://doi.org/10.1002/eji.201343751.

22. Wegdam-Blans MC, Kampschreur LM, Delsing CE, Bleeker-Rovers CP, Sprong T, van Kasteren ME, Notermans DW, Renders NH, Bijlmer HA, Lestrade PJ, Koopmans MP, Nabuurs-Franssen MH, Oosterheert JJ, Dutch Q. 2012. Chronic Q fever: review of the literature and a proposal of new diagnostic criteria. J Infect 64:247-259. https://doi .org/10.1016/j.jinf.2011.12.014.

23. Wielders CC, Boerman AW, Schimmer B, van den Brom R, Notermans DW, van der Hoek W, Schneeberger PM. 2015. Persistent high lgG phase I antibody levels against Coxiella burnetii among veterinarians compared to patients previously diagnosed with acute $\mathrm{Q}$ fever after three years of follow-up. PLoS One 10:e0116937. https://doi.org/10 .1371 /journal.pone.0116937.

24. Karagiannis I, Schimmer B, Van Lier A, Timen A, Schneeberger P, Van Rotterdam B, De Bruin A, Wijkmans C, Rietveld A, Van Duynhoven Y. 2009. Investigation of a $\mathrm{Q}$ fever outbreak in a rural area of The Netherlands. Epidemiol Infect 137:1283-1294. https://doi.org/10.1017/S095026 8808001908.

25. Morroy G, Van Der Hoek W, Nanver ZD, Schneeberger PM, BleekerRovers CP, Van Der Velden J, Coutinho RA. 2016. The health status of a village population, 7 years after a major $Q$ fever outbreak. Epidemiol Infect 144:1153-1162. https://doi.org/10.1017/S0950268815002472.

26. Moise L, Gutierrez A, Kibria F, Martin R, Tassone R, Liu R, Terry F, Martin B, De Groot AS. 2015. iVAX: an integrated toolkit for the selection and optimization of antigens and the design of epitope-driven vaccines. Hum Vaccin Immunother 11:2312-2321. https://doi.org/10.1080/21645515.2015 .1061159 .

27. Moise L, Gutierrez AH, Bailey-Kellogg C, Terry F, Leng Q, Abdel Hady KM, VerBerkmoes NC, Sztein MB, Losikoff PT, Martin WD, Rothman AL, De Groot AS. 2013. The two-faced T cell epitope: examining the host-microbe interface with JanusMatrix. Hum Vaccin Immunother 9:1577-1586. https://doi.org/10.4161/hv.24615. 